\title{
Health Literacy Correlates to HPV Vaccination Among US Adults Ages 27-45
}

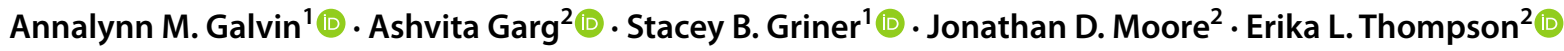

Accepted: 3 December 2021 / Published online: 13 January 2022

(c) The Author(s) under exclusive licence to American Association for Cancer Education 2021

\begin{abstract}
Human papillomavirus (HPV) vaccination is now available for adults aged $27-45$ as a shared clinical decision. Health literacy skills (i.e., accessing, understanding, appraising, applying information) may facilitate vaccine decision-making for adults with a provider recommendation. This study assessed associations between health literacy skills and willingness to get a provider-recommended HPV vaccine among newly eligible US adults. In 2020, US participants (51\% women), aged 27-45 years, were surveyed online $(n=691)$. The outcome was willingness (willing/not willing) to get the HPV vaccine with provider recommendation. Measures were adapted from Sørensen's multidimensional European Health Literacy Scale, which assesses health literacy among four domains (i.e., access, understanding, appraisal, application). Adjusted odds ratios were calculated for the outcome and each health literacy domain, adjusting for personal health determinants (e.g., age, sex). The sample consisted of primarily non-Hispanic (91.2\%), White (74.4\%), and married (60.7\%) adults. Approximately $65 \%$ of participants were willing to get a provider-recommended HPV vaccine. Higher willingness to vaccinate with provider recommendation was significantly associated with increased HPV knowledge (understanding; aOR $=1.13,95 \%$ CI 1.04, 1.24), ability to understand HPV information (understanding; $\mathrm{aOR}=1.96,95 \% \mathrm{CI} 1.09,3.52$ ), increased perceived vulnerability to HPV-related cancer (appraising; aOR $=3.22,95 \%$ CI 1.83, 5.69), and the need for more information on vaccine safety to seek vaccination (applying; aOR $=3.25 ; 95 \%$ CI $2.05,5.16$ ). Utilizing a multidimensional health literacy framework to evaluate facilitators to HPV vaccination uptake among adults aged 27-45 can help guide future interventions by targeting accurate, easy-to-understand HPV information that connects vaccination efficacy to reduction in HPV cancer risk.
\end{abstract}

Keywords Health literacy $\cdot$ HPV vaccination $\cdot$ Provider recommendation $\cdot$ Shared clinical decision

\section{Introduction}

Annalynn M. Galvin

annalynngalvin@my.unthsc.edu

Ashvita Garg

ashvitagarg@my.unthsc.edu

Stacey B. Griner

stacey.griner@unthsc.edu

Jonathan D. Moore

jonathanmoore@my.unthsc.edu

Erika L. Thompson

erika.thompson@unthsc.edu

1 Department of Health Behavior and Health Systems, University of North Texas Health Science Center, 3500 Camp Bowie Blvd., Fort Worth, TX 76107, USA

2 Department of Biostatistics and Epidemiology, University of North Texas Health Science Center, 3500 Camp Bowie Blvd., Fort Worth, TX, USA
Human papillomavirus (HPV), the most common sexually transmitted infection in the USA [1], causes six types of cancer and genital warts [2]. A key prevention strategy for HPV and HPV-related cancers is HPV vaccination. Currently, the Advisory Committee on Immunization Practices recommends routine vaccination for 11- and 12-year-olds in the USA. Unvaccinated persons can receive catch-up vaccination until age 26 [3]. As of 2019, adults ages 27-45 can also receive the HPV vaccine; however, routine vaccination is not recommended, and receipt depends on a shared clinical decision with their healthcare provider [3]. To translate this new HPV vaccine guideline into practice, we need to understand what contributes to HPV vaccination decisions in this age group. Preliminary research has shown that adults ages 27-45 years old are more likely to ask their provider about the vaccine or be likely to receive the HPV vaccine if 
they perceive a benefit to vaccination [4]. Thus, a key factor may be provider recommendation, as provider recommendation has been a key facilitator for HPV vaccination for other age groups [5].

To understand how provider recommendation may influence HPV vaccine uptake for adults ages $27-45$, it is important to assess patient information needs related to shared decision making. Within this age group, individuals reported they do not have enough information about the HPV vaccine to make a vaccination decision and specifically needed more information about the efficacy and safety of the vaccine [6]. However, addressing these information needs alone may not lead to vaccine uptake, but considering these needs in context with the preferred sources of information, how the information is processed, appraised, and used may provide a more complete picture [7]. As health care providers begin transitioning toward making these recommendations for their patients and implementing HPV vaccination shared decision making into practice, optimizing shared decision making and information seeking with qualified health care providers is critical. One way to potentially optimize shared decision making is through assessing the health literacy needs and assets for these newly eligible adults.

In the broadest sense, health literacy provides social and systems-level context on an individual's interactions with health information [7]. Health literacy is dynamic and amenable to improvement through active provider engagement [8]. Improved health literacy strengthens the connection between exposure to health information and engaging in health behavior based on said information [9]. After synthesizing several definitions of health literacy, four primary dimensions of health literacy that can serve as targets for intervention were identified: (1) access and obtain, (2) understand, (3) process and appraise, and (4) apply and use relevant health information [7]. Furthermore, this multidimensional model has been utilized in several studies to identify salient health literacy factors specifically for HPV and cervical cancer-related health promotion [8, 10, 11]. For adults aged 27-45 who are newly eligible for HPV vaccination with a shared clinical decision, further exploration of how the various dimensions of health literacy facilitate this information processing is needed.

Since adults aged $27-45$ potentially require increased health literacy to engage with providers to make the shared clinical decision for HPV vaccination, this study aims to evaluate associations between four domains of health literacy and willingness to get HPV vaccine if a provider recommends vaccination. It is hypothesized a priori that assessing various domains of health literacy will be more strongly associated with willingness to get HPV vaccine from a provider recommendation than personal determinants alone. Moreover, it is hypothesized a priori that there will be significant factors across multiple domains of health literacy (i.e., finding, understanding, accessing, applying) associated with getting an HPV vaccine with provider recommendation.

\section{Methods}

\section{Participants and Procedures}

Data for this study were drawn from a cross-sectional survey where participants aged 27-45 years with no history of HPV vaccination were recruited online $(n=691)$. Participant recruitment was conducted using the Qualtrics online panel, and Qualtrics provided compensation to the participants. Due to higher HPV-related disparities among nonHispanic Black and Hispanic populations [12], oversampling was performed for those groups. Recruitment was conducted between April and May 2020, and the study was approved by the North Texas Regional Institutional Review Board. Informed consent was obtained after the nature and possible consequences of the studies were fully detailed, prior to start of the survey.

\section{Measures}

The outcome of interest was willingness to receive the HPV vaccine with provider recommendation with the question asking, "How willing are you to get the HPV vaccine in the next six months if the doctor recommended it to you?" The response options were based on a 5-item Likert scale and ranged from "very willing" to "very unwilling." Based on the distribution of data, the variable was dichotomized into "willing" ("very willing," "willing") and "not willing" ("neither willing nor unwilling," "unwilling," "very unwilling").

Predictor variables were selected based on the Sørensen's multidimensional European Health Literacy Scale [13], which assesses health literacy among four domains (i.e., access, understanding, appraisal, application). For the access domain, to assess the ease of finding HPV vaccination information, participants were asked, "Please rate how easy it is for you to find information on HPV vaccination" with four response options ranging from "very easy" to "not easy," dichotomized into "easy" ("very easy," "easy") and "not easy" ("somewhat easy," "not easy"). The understand domain was assessed using two variables-ease of understanding HPV vaccination information and HPV knowledge score, based on a scale adapted from 2019 ACIP-recommended items to consider with shared decision-making: details on risk based on relationship status, sexual partnerships, and age, testing for HPV, efficacy of the vaccine, effectiveness of the vaccine, and prevention of HPV. Ease of understanding HPV vaccination information was assessed by asking, "Please rate how easy it is for you to understand information on HPV vaccination" with the four response 
options dichotomized to "easy" ("very easy," "easy") and "not easy" ("somewhat easy," "not easy"). HPV knowledge was assessed through a thirteen-item scale developed based on the recently modified HPV vaccine guidelines. The response options included "true," "false," and "don't know." Incorrect response and "don't know" options were coded as incorrect. Participants received 1-point for each correct response, and responses were summed to derive the total score for each participant. A higher score indicated higher knowledge, with potential scores ranging from 0 to 13. The appraisal domain was assessed by asking participants about their perceived vulnerability to genital warts and cancer without the HPV vaccine. The question asked, "Without the HPV vaccine, what do you think your chances are that you will get... (i) genital warts; (ii) cancer at some point in your life?" with five response options dichotomized as "likely" ("very likely," "likely") and "not likely" ("neither," "unlikely," "very unlikely"). The apply domain was assessed by asking, "How much do you agree... I need more information about how well the HPV vaccine would work for me before I decide whether to get it or not." The five response options were dichotomized to "agree" ("strongly agree," "agree") and "not agree" ("neither," "disagree," "strongly disagree").

Personal health determinants included age, sex (female, male), race (White, Black, multiracial, other), ethnicity (Hispanic, non-Hispanic), education (high school or less, some college/trade school/2-year degree, Bachelor's/4-year degree, graduate degree), insurance status (private, public, uninsured or don't know), and marital status (married, single, other).

\section{Data Analysis}

Univariate descriptive statistics were conducted for all variables. Bivariate cross-tabulation was assessed with health literacy variables and the outcome (willingness to get HPV vaccine with provider recommendation). Model fit statistics were assessed for separate nested models with the outcome. Models included personal health determinant covariates, health literacy domains, controlling for other variables in the model. This was completed to determine whether all domains of health literacy combined is more strongly associated with willingness to get HPV vaccine from a provider recommendation versus personal health determinants alone.

The base model analyzed the outcome variable with the demographic and socioeconomic covariates. Access variables were then added to the model, then understand variables, followed by appraisal variables, and finally application variables. Each time variables were added to the model, the new model was compared to the previous model using a likelihood ratio chi-squared test to examine whether the added covariates significantly improved model fit. For additional model fit analysis, Akaike information criterion (AIC) was also compared. AIC accounts for both, model goodness of fit and the simplicity of the model, mitigating overfitting and underfitting risks [14]. Lower AICs indicate better model fit. After establishing the final model, adjusted logistic regression analyzed the associations with willingness to get HPV vaccine with provider recommendation and all health literacy predictors, controlling for personal health determinants. All analyses were conducted using SAS 9.4 software.

\section{Results}

\section{Sample-Descriptive and Bivariate Statistics}

Table 1 presents personal health characteristics of the sample by willingness to vaccinate with provider recommendation. Overall, $65.3 \%$ of the 636 participants were willing to

Table 1 Sample personal health determinants by willingness to vaccinate for HPV with provider recommendation among participating US adults aged $27-45(n=691), 2020$

\begin{tabular}{|c|c|c|}
\hline & \multicolumn{2}{|c|}{$\begin{array}{l}\text { Willingness to vaccinate with pro- } \\
\text { vider recommendation }\end{array}$} \\
\hline & Willing $N(\%)$ & Unwilling $N(\%)$ \\
\hline $\mathrm{Age}^{\mathrm{a}}$ & $36.4(5.0)$ & $36.8(5.4)$ \\
\hline \multicolumn{3}{|l|}{ Sex assigned at birth } \\
\hline Male & $245(72.1)$ & 95 (27.9) \\
\hline Female & $190(54.1)$ & $161(45.9)$ \\
\hline \multicolumn{3}{|l|}{ Race } \\
\hline White & $328(64.2)$ & $183(35.8)$ \\
\hline Black & $28(46.7)$ & $32(53.3)$ \\
\hline Other & $43(63.2)$ & $25(36.8)$ \\
\hline Mixed race & $36(69.2)$ & $16(30.8)$ \\
\hline \multicolumn{3}{|l|}{ Hispanic ethnicity } \\
\hline Yes & $39(67.2)$ & $19(32.8)$ \\
\hline No & $396(62.6)$ & $237(37.4)$ \\
\hline \multicolumn{3}{|l|}{ Education level } \\
\hline High school degree or less & $65(46.4)$ & $75(53.6)$ \\
\hline $\begin{array}{l}\text { Some college/trade school/2- } \\
\text { year degree }\end{array}$ & $93(47.2)$ & $83(47.2)$ \\
\hline 4-year degree & $131(65.8)$ & $68(34.2)$ \\
\hline Graduate school & $146(83.0)$ & $30(17.1)$ \\
\hline \multicolumn{3}{|l|}{ Insurance status } \\
\hline Private insurance & $270(70.9)$ & $111(29.1)$ \\
\hline Public insurance & $124(59.1)$ & $86(41.0)$ \\
\hline None/don't know & $41(41.0)$ & $59(59.0)$ \\
\hline \multicolumn{3}{|l|}{ Marital status } \\
\hline Single & $124(55.1)$ & $101(44.9)$ \\
\hline Married & $280(68.8)$ & $127(31.2)$ \\
\hline Other & $31(52.5)$ & $28(47.5)$ \\
\hline
\end{tabular}

${ }^{\mathrm{a}}$ Mean, SD 
vaccinate with a provider recommendation. Approximately half of the sample were assigned male at birth $(49.2 \%)$, with an average age of 36.6 years $(\mathrm{SD}=5.1)$. Most participants identified as non-Hispanic (91.6\%), White (74.5\%), married (58.9\%), with private insurance (55.1\%). Education level was evenly distributed across the four categories, with most participants reporting Bachelor's/4-year degrees (28.8\%). Regarding personal health characteristics, significant $(p<0.05)$ bivariate associations with willing to vaccinate with a provider recommendation included age, sex assigned at birth, education level, marital status, and insurance status.

\section{Health Literacy-Descriptive Statistics}

Table 2 depicts the descriptive frequencies for willingness to vaccinate for the health literacy covariates. Many participants reported ease with finding (67.9\%) and understanding (65.6\%) HPV vaccination information versus participants who did not report ease in finding and understanding, respectively. Out of the possible 13 points, the average HPV knowledge score was $6.0(\mathrm{SD}=2.8)$. Most participants reported a likelihood of getting cancer $(67.9 \%)$ and genital warts (65.6\%) without the HPV vaccine compared to those who found these diagnoses unlikely. Lastly, most participants found it unlikely to get cancer (68.5\%) and genital warts $(72.1 \%)$ without the HPV vaccine versus a higher likelihood, indicating lower perceived vulnerability. For the unadjusted bivariate chi-square analysis (including $t$-tests for continuous HPV knowledge scores), willingness to vaccinate with provider recommendation was significantly associated with all health literacy covariates $(\mathrm{p}<0.05)$.

\section{Model Fit Statistics}

For model fit statistics, 5 different models were compared: (1) personal determinant covariates only $(-2 \log L=822$, $\mathrm{DF}=13, \mathrm{AIC}=850)$; (2) personal and access covariates $(-2 \log \mathrm{L}=800, \mathrm{DF}=14, \mathrm{AIC}=830)$; (3) personal, access, and understand covariates $(-2 \log \mathrm{L}=544, \mathrm{DF}=16$, $\mathrm{AIC}=578)$; (4) personal, access, understand, and appraise covariates $(-2 \log \mathrm{L}=514, \mathrm{DF}=18, \mathrm{AIC}=552)$; and (5) the full model-personal, access, understand, appraise, and apply covariates $(-2 \log \mathrm{L}=489, \mathrm{DF}=19, \mathrm{AIC}=528)$. Each model had progressively lower $-2 \log \mathrm{L}$ values (with statistically significant $[p<0.05]$ chi-square likelihood tests indicating that the larger model was better than the reduced model) and progressively lower AICs. Furthermore, the final model had the lowest AIC. Therefore, the adjusted logistic regression model for all health literacy and personal covariates were analyzed and interpreted (Table 3).

\section{Willingness to Receive HPV Vaccination with Provider Recommendation}

Table 3 displays the results from the final adjusted model for the associations between health literacy domains and willingness to receive the HPV vaccine after provider recommendation. Understand, appraise, and apply domains were significantly associated with willingness to receive HPV
Table 2 Descriptive frequencies and unadjusted $p$-values for health literacy covariates among US adults aged 27-45 $(n=691)$, 2020

\begin{tabular}{|c|c|c|c|}
\hline \multirow[t]{2}{*}{ Health literacy covariates } & \multicolumn{2}{|c|}{$\begin{array}{l}\text { Willingness to vaccinate with pro- } \\
\text { vider recommendation }\end{array}$} & \multirow[t]{2}{*}{$p$-value } \\
\hline & Willing $N(\%)$ & Unwilling $N(\%)$ & \\
\hline Access: Easy to find information & & & $<0.0001$ \\
\hline Easy & $329(70.2)$ & $140(29.9)$ & \\
\hline Not easy & $106(47.8)$ & $116(52.3)$ & \\
\hline Understand: Easy to understand information & & & $<0.0001$ \\
\hline Easy & $327(72.2)$ & $126(27.8)$ & \\
\hline Not easy & $108(45.4)$ & $130(54.6)$ & \\
\hline Understand: HPV knowledge ${ }^{a}$ & $6.41(2.40)$ & $5.14(3.24)$ & $<0.0001$ \\
\hline Appraise: Perceived vulnerability to cancer & & & $<0.0001$ \\
\hline Likely & $183(83.9)$ & $35(16.1)$ & \\
\hline Unlikely & $252(53.3)$ & $221(46.7)$ & \\
\hline Appraise: Perceived vulnerability to genital warts & & & $<0.0001$ \\
\hline Likely & $158(81.9)$ & $35(18.1)$ & \\
\hline Unlikely & $277(55.6)$ & $221(44.4)$ & \\
\hline Apply: Needs more safety info before making decision & & & $<0.0001$ \\
\hline Agree & $85(35.9)$ & $152(64.1)$ & \\
\hline Disagree & $350(77.1)$ & $104(22.9)$ & \\
\hline
\end{tabular}

${ }^{\mathrm{a}}$ Mean, SD; higher scores $=$ more knowledge, range $=0-13$ 
Table 3 Adjusted odds ratio associations between four health literacy domains and willingness to get an HPV vaccination after a provider recommendation for US adults aged 27-45 $(n=691), 2020$
Health literacy covariates

Willingness to vaccinate with provider recommendation

\begin{tabular}{|c|c|}
\hline \multicolumn{2}{|l|}{ Access: Easy to find information } \\
\hline Easy & $0.92(0.51,1.66)$ \\
\hline Not easy & Reference \\
\hline \multicolumn{2}{|l|}{ Understand: Easy to understand information } \\
\hline Easy & $1.96(1.09,3.52)^{*}$ \\
\hline Not easy & Reference \\
\hline Understand: HPV knowledge & $1.13(1.04,1.24)^{*}$ \\
\hline \multicolumn{2}{|l|}{ Appraise: Perceived vulnerability to cancer } \\
\hline Likely & $3.22(1.83,5.69)^{*}$ \\
\hline Unlikely & Reference \\
\hline \multicolumn{2}{|c|}{ Appraise: Perceived vulnerability to genital warts } \\
\hline Likely & $1.31(0.70,2.46)$ \\
\hline Unlikely & Reference \\
\hline \multicolumn{2}{|c|}{ Apply: Needs more safety info before making decision } \\
\hline Agree & $3.25(2.05,5.16)^{*}$ \\
\hline Disagree & Reference \\
\hline \multicolumn{2}{|l|}{ Personal health determinants covariates } \\
\hline Age & $0.98(0.94,1.03)$ \\
\hline \multicolumn{2}{|l|}{ Sex assigned at birth } \\
\hline Female & Reference \\
\hline Male & $2.66(1.63,4.35)^{*}$ \\
\hline \multicolumn{2}{|l|}{ Race } \\
\hline White & Reference \\
\hline Black & $0.83(0.36,1.94)$ \\
\hline Other & $1.18(0.49,2.83)$ \\
\hline Multiracial & $1.09(0.46,2.57)$ \\
\hline \multicolumn{2}{|l|}{ Hispanic ethnicity } \\
\hline Non-Hispanic & $0.92(0.41,2.06)$ \\
\hline Hispanic & Reference \\
\hline \multicolumn{2}{|l|}{ Education level } \\
\hline HS or less & $1.20(0.63,2.30)$ \\
\hline Some college/trade school/2-year degree & Reference \\
\hline Bachelor's degree & $1.33(0.74,2.37)$ \\
\hline Graduate school & $2.13(1.07,4.24)^{*}$ \\
\hline \multicolumn{2}{|l|}{ Insurance status } \\
\hline Private insurance & Reference \\
\hline Public insurance & $1.12(0.66,1.90)$ \\
\hline None/don't know & $0.67(0.33,1.37)$ \\
\hline \multicolumn{2}{|l|}{ Marital status } \\
\hline Single & Reference \\
\hline Married & $1.21(0.71,2.04)$ \\
\hline Other & $1.12(0.50,2.50)$ \\
\hline
\end{tabular}

${ }^{*} p$-value $<0.05$

vaccine. Participants who found vaccine information easy to understand had almost twice the odds of being willing to vaccinate compared to participants who did not find the information easy to understand (aOR $=1.96$; 95\% CI 1.09 , 3.52). With a one-unit increase in HPV knowledge score, the odds of being willing to vaccinate increased $(\mathrm{aOR}=1.13$; 95\% CI 1.04, 1.24). Additionally, participants who were more likely to have perceived vulnerability to cancer $(\mathrm{aOR}=3.22 ; 95 \% \mathrm{CI} 1.83,5.69)$ and agreed that they needed more safety information before deciding $(\mathrm{aOR}=3.25 ; 95 \%$ 
CI 2.05, 5.16) had higher odds of being willing to vaccinate with a provider recommendation compared to participants with less perceived vulnerability to cancer and participants who did not need more safety information before deciding to vaccinate, respectively. Among the personal health determinants, males $(\mathrm{aOR}=2.66 ; 95 \%$ CI $1.63,4.35)$ compared to females and participants with graduate school education $(\mathrm{aOR}=2.13 ; 95 \%$ CI 1.07, 4.24) compared to high school graduates had higher odds of being willing to vaccinate with provider recommendation.

\section{Discussion}

Due to the recent guideline change recommending the administration of the HPV vaccine to adults ages 27-45 years based on provider recommendation, we sought to assess associations between health literacy and willingness to receive the HPV vaccine with a provider recommendation among these recently eligible US adults. In this study, sex assigned at birth and education level were associated with the willingness to receive the HPV vaccine based on a provider recommendation. Furthermore, while access to HPV vaccination information was no longer significant when adjusting for all four domains of health literacy, a patient's willingness to receive the HPV vaccine based on a provider recommendation was associated with covariates that measured understanding, appraisal, and application of health information.

Since education level and ability to interpret and have a dialogue with a provider are critical, it is important to note that participants with graduate education had significantly higher odds of being willing to get the HPV vaccine with provider recommendation. One study that assessed HPV vaccination attitudes across high school, college, and graduate level students found no association between education level and HPV vaccine series completion [15]. Differences in findings could be attributed to the different age groups since the former study excluded adults over 26 years [15]. Moreover, participants assigned male at birth had a much higher odds of willingness to receive an HPV vaccine with provider recommendation, compared to those assigned female at birth. Similarly, in a study consisting of national sample of adolescent males, receiving a provider recommendation had over eight times the odds of initiating the HPV vaccination series than no recommendation [16]. Previous evidence highlights the disproportionately higher rates of HPV vaccine provider communication [17] and HPV vaccination completion [18] for women populations. In support of the higher odds of willingness to vaccinate among male participants, further research is needed to identify whether provider recommendation can serve as a potential avenue in increasing HPV vaccination rates in males generally as well as males who fall within the newer guideline ages of $27-45$.

Within the understand domain, knowledge and perceived ease of understanding HPV vaccine information was statistically associated with willingness to vaccinate with a provider recommendation, yet the participant's ability to find information about HPV vaccination (e.g., the access domain) was not. The findings for this study may be a result of improved accessibility to health information based on increased health information exposure on the internet and social media [19], as well as the survey collection via online sampling. Similarly, among a US sample of women aged 30-65, accessibility of cervical cancer information was not statistically significant yet cervical cancer knowledge and ability to understand cervical cancer information was significant [11]. A recent study assessing university students' HPV knowledge, vaccination status, and health literacy found significant associations between health literacy and HPV knowledge as well as health literacy and HPV vaccination status [20]. The current study supports on these findings, with a more robust health literacy assessment and newly eligible adults aged 27-45. The potential for augmenting or hindering a newly eligible adult's capacity to understand HPV knowledge and engage in a conversation with their provider about the HPV vaccine is critical. Future studies should account for significant health literacy skills regarding how easily people can understand HPV vaccination information accessed before provider conversations about HPV vaccination.

Within the appraisal domain of health literacy, perceived vulnerability to cancer was a significant correlate to HPV vaccination with a provider recommendation. A previous study found that adults 27-45 who perceived a benefit from HPV vaccination would be more likely to ask their healthcare provider about the vaccine [4]. As such, these two findings may highlight an underlying phenomenon that persons who perceived they are vulnerable to cancer may characterize this risk as a potential benefit if vaccinated. Early work examining young adults' beliefs about HPV and cancer also found a connection between perceived vulnerability and HPV vaccination [21]. While the present study found perceived vulnerability for cancer to be associated with vaccination intention, perceived vulnerability for genital warts was not present. Thus, adults may be considering the more severe health outcome in their appraisal assessment.

In the application domain, people who needed more safety information had higher odds of being willing to vaccinate with provider recommendation, compared to people who disagreed with needing more safety information before making an HPV vaccination decision. This finding suggests that among those with limited information on vaccine safety, provider recommendation may play a stronger role than those who are less knowledgeable. A National Immunization 
Survey-Teen study of parents with no intention to provide HPV vaccination to their adolescent daughters found that $18 \%$ of these parents reported safety and efficacy concerns [22]. Among 27- to 45-year-olds, most prefer to make vaccine decisions alone or in collaboration with their healthcare provider, and the results from this study align with previous data indicating a large proportion of individuals (ages 27-45) reported a need for more information about vaccine safety and efficacy prior to deciding to vaccinate [6]. Provider recommendation has been noted as a key predictor of HPV vaccination [23], and providers are encouraged to reframe not getting a vaccination as a decision, comparing safety risks with not getting the HPV vaccine to the minimal risks with obtaining the vaccine [24]. As message content from providers often includes information about vaccine efficacy and safety, a more in-depth examination of this complex relationship may be needed, especially in light of recent COVID-19 vaccination discussions about how safety and efficacy are determined [25].

\section{Limitations}

The current study has a few limitations to consider. Due to the cross-sectional design of the data collection, temporal sequence and causal inference could not be determined. Additionally, since the survey was collected online and was only written in the English language, those lacking adequate computer/Internet access and non-English speaking individuals were not included in the sample, which may introduce some selection bias. There is also a lack of language and acculturation information in the data collected, limiting our understanding of how these factors may have affected the results of the study. Although strongly associated, "willingness to get the HPV vaccine" does not definitively mean that the participants will get the HPV vaccine. Further research measuring actual vaccination behavior would be necessary to determine if these health literacy constructs provide a valid model for vaccination behavior. Furthermore, because the data collected were self-report, there may be misclassification due to social desirability bias or misunderstanding of survey questions.

\section{Conclusion}

Despite these limitations, this study evaluates health literacy and willingness to get an HPV vaccination with provider recommendation among a sample of US adults aged 27-45 who are eligible for HPV vaccination with shared clinical decision. Utilizing a multidimensional health literacy framework to evaluate barriers to and facilitators of HPV vaccination uptake among adults aged $27-45$ barriers can help guide future interventions by targeting accurate, easy-to-understand HPV information that connects vaccination efficacy to reduction in HPV cancer risk.

Author Contribution Annalynn M. Galvin: Conceptualization, methodology, formal analysis, writing — original draft, writing — review and editing, visualization; Ashvita Garg: Writing—original draft, writingreview and editing; Stacey B. Griner: Writing-original draft, writing — review and editing; Jonathan D. Moore: Writing—original draft, writing - review and editing; Erika L. Thompson: Conceptualization, methodology, writing_original draft, writing—review and editing.

Data Availability Not available.

Code Availability Not available.

\section{Declarations}

Ethics Approval The authors and project followed all ethical guidelines observed by the North Texas Regional Institutional Review Board.

Consent to Participate Informed consent was obtained from all individual participants included in the study.

Consent for Publication N/A.

Conflict of Interest Author Erika Thompson is a consultant to Merck. The other authors declare no competing interests.

\section{References}

1. Kreisel KM, Spicknall IH, Gargano JW, Lewis FMT, Lewis RM, Markowitz LE, Roberts H, Johnson AS, Song R, St Cyr SB, Weston EJ, Torrone EA, Weinstock HS (2021) Sexually transmitted infections among US women and men: prevalence and incidence estimates, 2018. Sex Transm Dis 48(4):208-214. https://doi.org/ 10.1097/OLQ.0000000000001355

2. Senkomago V, Henley SJ, Thomas CC, Mix JM, Markowitz LE, Saraiya M (2019) Human papillomavirus-attributable cancers - United States, 2012-2016. MMWR Morb Mortal Wkly Rep 68(33):724-728. https://doi.org/10.15585/mmwr.mm683 $3 \mathrm{a} 3$

3. Meites E, Szilagyi PG, Chesson HW, Unger ER, Romero JR, Markowitz LE (2019) Human papillomavirus vaccination for adults: updated recommendations of the Advisory Committee on Immunization Practices. MMWR Morb Mortal Wkly Rep 68(32):698-702. https://doi.org/10.15585/mmwr.mm6832a3

4. Thompson EL, Garg A, Galvin AM, Moore JD, Kasting ML, Wheldon CW (2021) Correlates of HPV vaccination intentions among adults ages $27-45$ years old in the U.S. J Community Health 46(5):893-902. https://doi.org/10.1007/ s10900-021-00968-3

5. Oh NL, Biddell CB, Rhodes BE, Brewer NT (2021) Provider communication and HPV vaccine uptake: a meta-analysis and systematic review. Prev Med 148:106554. https://doi.org/10.1016/j. ypmed.2021.106554

6. Wheldon CW, Garg A, Galvin AM, Moore JD, Thompson EL (2021) Decision support needs for shared clinical decision-making regarding HPV vaccination among adults $27-45$ years of age. 
Patient Educ Couns S0738-3991(21):00281. https://doi.org/10. 1016/j.pec.2021.04.016

7. Sørensen K, Van den Broucke S, Fullam J, Doyle G, Pelikan J, Slonska Z, Brand H (2012) (HLS-EU) Consortium Health Literacy Project European. Health literacy and public health: a systematic review and integration of definitions and models. BMC Public Health 12:80. https://doi.org/10.1186/1471-2458-12-80

8. Best AL, Vamos C, Choi SK, Thompson EL, Daley E, Friedman DB (2017) Increasing routine cancer screening among underserved populations through effective communication strategies: application of a health literacy framework. J Cancer Educ 32(2):213-217. https://doi.org/10.1007/s13187-017-1194-7

9. Nutbeam D (2008) The evolving concept of health literacy. Soc Sci Med 67(12):2072-2078. https://doi.org/10.1016/j.socscimed. 2008.09.050

10. Thompson EL, Wheldon CW, Vamos CA, Griner SB, Daley EM (2019) How is health literacy related to Pap testing among US women? J Cancer Educ 34(4):789-795. https://doi.org/10.1007/ s13187-018-1375-Z

11. Galvin AM, Garg A, Matthes S, Thompson EL (2021) Utilizing a multidimensional health literacy framework to assess cervical cancer screening nonadherence. Health Educ Behav 48(5):710718. https://doi.org/10.1177/10901981211001851

12. U.S. Cancer Statistics Working Group. U.S. Cancer Statistics Data Visualization Tool. https://gis.cdc.gov/Cancer/USCS/DataViz. html ;2021 [accessed 15 Mar 2021]

13. Sørensen K, Van den Broucke S, Pelikan JM, Fullam J, Doyle G, Slonska Z, Kondilis B, Stoffels V, Osborne RH, Brand H (2013) HLS-EU Consortium. Measuring health literacy in populations: illuminating the design and development process of the European Health Literacy Survey Questionnaire (HLS-EU-Q). BMC Public Health 13:948. https://doi.org/10.1186/1471-2458-13-948

14. Akaike H (1974) A new look at the statistical model identification. IEEE Trans Autom Control 19(6):716-723. https://doi.org/ 10.1109/TAC.1974.1100705

15. Suryadevara M, Bonville JR, Kline RM, Magowan C, Domachowske E, Cibula DA, Domachowske JB (2016) Student HPV vaccine attitudes and vaccine completion by education level. Hum Vaccin Immunother 12(6):1491-1497. https://doi.org/10.1080/ 21645515.2015.1123359

16. Landis K, Bednarczyk RA, Gaydos LM (2018) Correlates of HPV vaccine initiation and provider recommendation among male adolescents, 2014 NIS-Teen. Vaccine 36(24):3498-3504. https://doi. org/10.1016/j.vaccine.2018.04.075

17. McRee AL, Gilkey MB, Dempsey AF (2014) HPV vaccine hesitancy: findings from a statewide survey of health care providers.
J Pediatr Health Care 28(6):541-549. https://doi.org/10.1016/j. pedhc.2014.05.003

18. Stokley S, Jeyarajah J, Yankey D, Cano M, Gee J, Roark J, Curtis RC, Markowitz L (2014) Immunization Services Division, National Center for Immunization and Respiratory Diseases, CDC; Centers for Disease Control and Prevention (CDC). Human papillomavirus vaccination coverage among adolescents, 2007-2013, and postlicensure vaccine safety monitoring, 2006-2014--United States. MMWR Morb Mortal Wkly Rep 63(29):620-624

19. Dunn AG, Surian D, Leask J, Dey A, Mandl KD, Coiera E (2017) Mapping information exposure on social media to explain differences in HPV vaccine coverage in the United States. Vaccine 35(23):3033-3040. https://doi.org/10.1016/j.vaccine.2017.04.060

20. Kitur H, Horowitz AM, Beck K, Wang MQ. 2021 HPV knowledge, vaccine status, and health literacy among university students. J Cancer Educ 1-8. https://doi.org/10.1007/s13187-021-01997-1

21. Patel DA, Zochowski M, Peterman S, Dempsey AF, Ernst S, Dalton VK (2012) Human papillomavirus vaccine intent and uptake among female college students. J Am Coll Health 60(2):151-161. https://doi.org/10.1080/07448481.2011.580028

22. Cheruvu VK, Bhatta MP, Drinkard LN (2017) Factors associated with parental reasons for "no-intent" to vaccinate female adolescents with human papillomavirus vaccine: National Immunization Survey - Teen 2008-2012. BMC Pediatr 17(1):52. https://doi.org/ 10.1186/s12887-017-0804-1

23. Gilkey MB, McRee AL (2016) Provider communication about HPV vaccination: a systematic review. Hum Vaccin Immunother 12(6):1454-1468. https://doi.org/10.1080/21645515.2015.11290 90

24. Zimet GD, Rosberger Z, Fisher WA, Perez S, Stupiansky NW (2013) Beliefs, behaviors and HPV vaccine: correcting the myths and the misinformation. Prev Med 57(5):414-418. https://doi.org/ 10.1016/j.ypmed.2013.05.013

25. Goodman JL, Grabenstein JD, Braun MM (2020) Answering key questions about COVID-19 vaccines. JAMA 324(20):2027-2028. https://doi.org/10.1001/jama.2020.20590

Publisher's Note Springer Nature remains neutral with regard to jurisdictional claims in published maps and institutional affiliations. 